\title{
Down-regulation of microRNA-155 suppressed Candida albicans induced acute lung injury by activating SOCS1 and inhibiting inflammation response
}

\author{
Xiaohua Li ${ }^{1,2 \dagger}$, Yuanzhong Gong ${ }^{3 \dagger}$, Xin Lin ${ }^{1}$, \\ Qiong $\operatorname{Lin}^{1}$, Jianxiong Luo ${ }^{1}$, Tianxing $\mathrm{Yu}^{1}$, \\ Junping $\mathrm{Xu}^{1}$, Lifang Chen ${ }^{1}$, Liyu $\mathrm{Xu}^{1 \star}$, \\ and Ying $\mathrm{Hu}^{1 \star}$ \\ ${ }^{1}$ Department of Pulmonary and Critical Care Medicine, Affiliated \\ Fuzhou First Hospital of Fujian Medical University, Fuzhou, \\ Fujian 350009, P. R. China \\ ${ }^{2}$ Department of Pulmonary and Critical Care Medicine, 900 Hospital of \\ the Joint Logistics Team, Fuzhou, Fujian 350001, P. R. China \\ ${ }^{3}$ Department of Respiratory Medicine, Nanping First Hospital Affiliated \\ to Fujian Medical University Nanping City, Fujian 353006, P. R. China \\ (Received Dec 23, 2021 / Revised Jan 3, 2022 / Accepted Jan 4, 2022)
}

Acute lung injury caused by Candida albicans could result in high mortality and morbidity. MicroRNA-155 (miR-155) and suppressor of cytokine signaling 1 (SOCS1) have been believed to play a key in the regulation of inflammatory response. Whether miR-155/SOCS1 axis could regulate the acute lung injury caused by $C$. albicans has not been reported. The acute lung injury animal model was established with acute infection of C. albicans. miR-155 inhibitor, miR-155 mimic, and sh-SOCS1 were constructed. The binding site between miR155 and SOCS1 was identified with dual luciferase reporter assay. Knockdown of miR-155 markedly inhibited the germ tube formation of $C$. albicans. Knockdown of miR-155 significantly up-regulated the expression of SOCS1, and the binding site between miR-155 and SOCS1 was identified. Knockdown of miR-155 improved the acute lung injury, suppressed inflammatory factors and fungus loading through SOCS1. Knockdown of SOCS1 greatly reversed the influence of miR155 inhibitor on the cell apoptosis in vitro. The improvement of acute lung injury caused by $C$. albicans, suppression of inflammatory response and C. albicans infection, and inhibitor of cell apoptosis were achieved by knocking down miR-155 through SOCS1. This research might provide a new thought for the prevention and treatment of acute lung injury caused by C. albicans through targeting miR-155/SOCS1 axis.

Keywords: Candida albicans, miR-155, SOCS1, acute lung injury

\footnotetext{
${ }^{\dagger}$ These authors contributed equally to this work.

${ }^{*}$ For correspondence. (L. Xu) E-mail: langzhongxu@163.com; Tel.: +8615880004509 / (Y. Hu) E-mail: sunnyhuying@163.com; Tel.: +86-137994 38035

Copyright (C) 2022, The Microbiological Society of Korea
}

\section{Introduction}

Acute lung injury characterized by severe inflammatory response, and it is mainly caused by infection (Yan et al., 2021). Currently, some therapeutic methods including glucocorticoids, surfactant, and nitric oxide have been used to treat acute lung injury patients. However, the mortality and morbidity in critically ill acute lung injury patients still remain high (Xu et al., 2021). Therefore, it is necessary to explore novel therapeutic agents for the treatment of acute lung injury.

Candida albicans is a common conditional pathogenic fungus. Remarkable increase of proliferation and enhancement of virulence could be observed when the immune function is decreased greatly (Vila et al., 2020; Li et al., 2021). The infection caused by C. albicans can be life-threatening. Currently, the commonly used drugs for the treatment of C. albicans infection are antibiotics. Due to drug resistance and adverse drug reactions, most antifungal drugs often cannot achieve desired therapeutic effect, and the mortality of invasive fungal infection remains high (Kobayashi-Sakamoto et al., 2018; Nidhi et al., 2020). Therefore, the development of new antifungal drugs is urgent.

MicroRNA (miRNA) is an endogenous non-coding micro$\mathrm{RNA}$, and they could bind to the $3^{\prime}$ noncoding region of the target gene mRNA, induce mRNA degradation or hinder its translation (Hassan et al., 2021). MiRNAs are composed of 19-24 nucleotides, with a length change of 1-2 bases at the $3^{\prime}$ end. miRNAs play a key role in regulating down-stream gene expression, and they have been reported to be widely involved in cell differentiation, proliferation, and apoptosis, which is closely related to tumor growth, lung injury and other diseases (Kittelmann and McGregor, 2019). MicroRNA-155 (miR-155) is located at human chromosome 21, and it has been believed to be widely involved in the inflammatory response (Mahernia et al., 2021). It was reported that miR-155 played a pro-inflammatory effect during regulating macrophages after C. albicans infection (Agustinho et al., 2017). miR-155 could regulate the balance between M1/M2 and further affect the function of macrophages. Meanwhile, miR-155 could negatively regulate the $\mathrm{M} 2 /$ pro-TH2 phenotype of macrophages by targeting IL-13 dependent factors (SOCS1 and CD 18). In addition, miR-155 was closely involved in inflammation-induced hippocampal neurogenic dysfunction (Woodbury et al., 2015). Knockdown of miR-155 could improve functional recovery and brain microvasculature growth, but inhibit brain tissue damage in the ischemia animal model (Pena-Philippides et al., 2016). However, if miR-155 could influence acute lung injury has not been reported. 
Suppressor of cytokine signaling (SOCS) is a family of immunosuppressive molecules. They are negative regulatory factors secreted by cells but can feedback inhibit cytokine signal transduction (Sun et al., 2013). SOCS can regulate a variety of cytokines and participate in the process of different inflammatory diseases. Suppressor of cytokine signaling 1 (SOCS1), the target gene of miR-155, is closely related to inflammatory response. SOCS1 is an important inhibitor factor of interferon and toll like receptor mediated response in various immune cells (Gingras et al., 2004). Previous report indicated that SOCS1 knockout mice developed severe inflammation 3 weeks after birth and eventually died of multiple organ dysfunction syndrome (Chikuma et al., 2017). It was reported that miR-155/SOCS1 axis was closely linked with lipopolysaccharide (LPS) (Cheng et al., 2012) and virus (Wang et al., 2010) induced macrophage inflammatory response. Meanwhile, miR-155 can target and regulate SOCS1 gene, enhance the immunomodulatory function of bone marrow mesenchymal stem cells (Zhang et al., 2018), and improve the tolerance of microglia of central nervous system to endotoxin (Sun et al., 2018). However, if miR-155/SOCS1 axis is involved in the regulation of acute lung injury has not been reported. In this study, we established acute lung injury through $C$. albicans infection, and constructed miR-155 inhibitor, miR155 mimic, and sh-SOCS1. Whether miR-155 could regulate acute lung injury through targeting SOCS1 was investigated. We firstly reported that down-regulation of miRNA-155 could inhibit $C$. albicans induced acute lung injury by activating SOCS1 and inhibiting inflammation response, which expands the potential application of miRNA-155. This study might provide a new therapeutic thought for the treatment of acute lung injury caused by C. albicans.

\section{Materials and Methods}

\section{Cell culture and LPS treatment}

MH-S cell line (Mice alveolar macrophages, Procell) was used in this study for in vitro research. The cells were cultured with RPMI-1640 medium containing 10\% FBS and $0.1 \mathrm{mM} \beta$ mercaptoethanol. The cells were cultivated in an incubator with $5 \% \mathrm{CO}_{2}$ and $37^{\circ} \mathrm{C}$. To establish acute lung injury cell model, LPS $(1 \mu \mathrm{g} / \mathrm{ml})$ was used to cultivate cell for $24 \mathrm{~h}$.

\section{Time-kill curve}

The constructed miR-155 inhibitor (Final concentration: 20 $\mu \mathrm{M})$ was mixed with prepared C. albicans solution $\left(2 \times 10^{3}\right.$ $\mathrm{CFU} / \mathrm{ml}$ ) in an incubator at $37^{\circ} \mathrm{C}$. After $0,4,8$, and $16 \mathrm{~h}$ incubation, the culture medium was coated on the sabouraud dextrose agar plate at $37^{\circ} \mathrm{C}$. After another $24 \mathrm{~h}$ incubation at $37^{\circ} \mathrm{C}$, the colony formations were counted.

\section{Germ tube formation}

Candida albicans $\left(5 \times 10^{6} \mathrm{CFU} / \mathrm{ml}\right)$ was coated into RPMI1640 medium containing 5\% FBS. miR-155 inhibitor (Final concentration: $20 \mu \mathrm{M}$ ), miR-155 mimics (Final concentration: $20 \mu \mathrm{M}$ ), and Itraconazole (Final concentration: $0.1 \mathrm{mg} / \mathrm{ml}$ ) were supplemented, and cultured using constant temperature oscillation $\left(180 \mathrm{~g} / \mathrm{min}, 37^{\circ} \mathrm{C}\right)$ for $4 \mathrm{~h}$. The mixed culture me- dium $(100 \mu \mathrm{l})$ was inoculated on the glass slide. The germ tubes were observed and counted with a microscope.

\section{Immunofluorescence staining}

The collected tissues were fixed with paraformaldehyde (4\%) for 48, and embedded with optimal cutting temperature compound (OCT, Sigma). The $10 \mu \mathrm{m}$ crosswise tissue sections were made for immunofluorescence and HE staining. After antigen repair, the tissues were treated with $5 \%$ bovine serum albumin for blocking. The tissues were incubated with anti-SOCS1 (1:1000, \#ab9870, Goat polyclonal to SOCS1, Abcam) antibody at $4^{\circ} \mathrm{C}$ for $12 \mathrm{~h}$. The tissues were washed with PBS 3 times, and incubated with second antibody (1:2000, Donkey polyclonal secondary antibody to goat IgG, \#ab150129, Abcam) for $2 \mathrm{~h}$. Cell nuclei was stained using DAPI. The fluorescent images were analyzed using a confocal microscope.

\section{Animal experiments}

Animal experiments were approved by the Animal Care and Use Committee of the Affiliated Fuzhou First Hospital of Fujian Medical University (20210014). BALB/c mice (Male, 9-10 weeks) purchased from Charles River were used in this research. The animal experiments were performed based on previous references (Patterson et al., 1993; Gamaletsou et al., 2019) and our own pre-experiment. The animal experiments were divided into the following groups: Control, C. albicans, C. albicans + miR-155 inhibitor, C. albicans + miR-155 mimics, C. albicans + Itraconazole, C. albicans + sh-SOCS1. Six animals were used in each group. The animals in the group control were intratracheally injected with $100 \mu \mathrm{l}$ PBS. The animals in other groups were intratracheally injected with C. albicans $\left(1 \times 10^{7} \mathrm{CFU}\right.$ in $\left.50 \mu \mathrm{l} \mathrm{PBS}\right)$ combined with miR155 inhibitor $(10 \mu \mathrm{g} / \mu \mathrm{l}$ in $50 \mu \mathrm{l} \mathrm{PBS}), \mathrm{miR}-155$ mimics $(10$ $\mu \mathrm{g} / \mu \mathrm{l}$ in $50 \mu \mathrm{l}$ PBS $)$ Itraconazole $(15 \mathrm{mg} / \mathrm{kg}$ in $50 \mu \mathrm{l} \mathrm{PBS})$, or sh-SOCS1 $(10 \mu \mathrm{g} / \mu \mathrm{l}$ in $50 \mu \mathrm{l}$ PBS). The animals were euthanized using $\mathrm{CO}_{2}$ inhalation $48 \mathrm{~h}$ after treatment. The blood, lung, liver, and kidney tissues were collected. The following items including temperature, inoculation sites, survival status, hair, and body change were monitored. Rapid weight loss (more than 20\%), rapid temperature decrease (more than $6^{\circ} \mathrm{C}$ ), incurable long-term diarrhea, and continuous lying down were set as the humane end points of animal experiment.

\section{Measurement of inflammatory factors}

The blood was collected from posterior orbital venous plexus of animals. The collected blood was centrifuged $(2,000 \mathrm{~g} / \mathrm{min}$, $4^{\circ} \mathrm{C}$ ) for $10 \mathrm{~min}$. The collected serum was stored at $-80^{\circ} \mathrm{C}$ before use. ELISA method was used to measure the levels of TNF- $\beta$ (\#PT903), IL-6 (\#PI335), TNF- $\alpha$ (\#PT516), and IL-10 (\#PI525) in the serum of animals. All ELISA kits were obtained from Beyotime.

\section{Measurement of $C$. albicans loading in different tissues}

After sacrifice, the tissues (kidney, liver, and lung) were collected and homogenized. The homogenized tissues were diluted 10 times, and coated on the sabouraud dextrose agar medium at $37^{\circ} \mathrm{C}$. After incubation for 2 days, the colony 
forming numbers were counted.

\section{Hematoxylin and eosin (HE) staining}

The following steps were performed for HE staining: Hematoxylin staining $(30 \mathrm{sec})$, washing with water $(15 \mathrm{sec})$, differentiation ( $5 \mathrm{sec}$ ) with $\mathrm{HCl} / 95 \%$ alcohol (1:100), washing with water (40 $\mathrm{min})$, eosin staining $(10 \mathrm{sec})$, washing with water (10 min). The sections were mounted with neutral gum, and captured with Zeiss AxioVision (Jena).

\section{Dual luciferase reporter assay}

Cells were cultured in a 24 well plate. Lipofectamine 2000 (Invitrogen life technologies) was used for co-transfection. Co-transfection with pmirGLO-SOCS1-WT or pmirGLOSOCS1-MUT and miR-155 mimic was performed. Dualluciferase reporter gene assay kit was used to measuring luciferase activity $48 \mathrm{~h}$ after transfection.

\section{qRT-PCR}

Trizol reagent (TaKaRa) was used to extract RNA, and cDNA was amplified using using RT-PCR with ChamQ $^{\mathrm{TM}}$ SYBR $^{\circledR}$ qPCR Master Mix (Vazyme). The primers used in this study was listed as follows: miR-155 (F: ATGCTAATC
GTGATAGGGG, R: GTGCAGGGTCCGAGGTAT); SOCS1 (F: AGCTCCTTCCCCTTCCAGATT, R: CCACATGGTT CCAGGCAAGTA); GAPDH (F: ATGGGGAAGGTGAA GGTCG, R: TCGGGGTCATTGATGGCAACAATA). $2^{-\Delta \Delta C T}$ method was applied to analyze results. The thermocycling protocol of qRT-PCR was listed below: $95^{\circ} \mathrm{C}(30 \mathrm{sec})$ and 40 cycles $\left(95^{\circ} \mathrm{C}, 3 \mathrm{sec}\right.$, and $\left.60^{\circ} \mathrm{C}, 30 \mathrm{sec}\right)$.

\section{Cell transfection}

The sh-SOCS1, miR-155 mimic, and miR-155 inhibitor were designed and synthesized by GenePharma. The cells were cultured in an incubator with $5 \% \mathrm{CO}_{2}$ and $37^{\circ} \mathrm{C}$. After reaching $70 \%$ confluence, transfection was preformed using Lipofectamine 2000 (\#11668027, Invitrogen).

\section{Flow cytometry}

Cells were seeded at 12 well plate, and cultured in the incubator with $5 \% \mathrm{CO}_{2}$ and $37^{\circ} \mathrm{C}$ for $24 \mathrm{~h}$. After treatment with LPS $(1 \mu \mathrm{g} / \mathrm{ml})$, miR-155 inhibitor, and sh-SOCS1 for $48 \mathrm{~h}$, cells were digested with trypsin. The collected cells were cultured with propidium iodide and Annexin V-FITC for 15 min in the dark. Cell apoptosis was analyzed using Cytomics ${ }^{\mathrm{TM}}$ FC500 Flow Cytometer (Beckman).
(A)

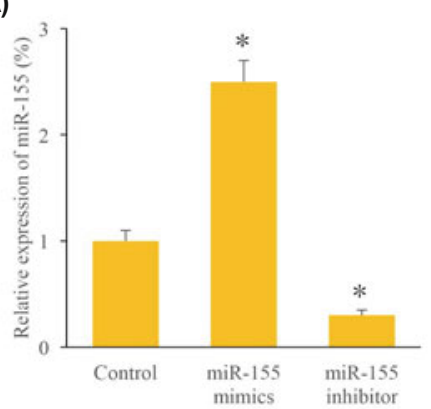

(C)

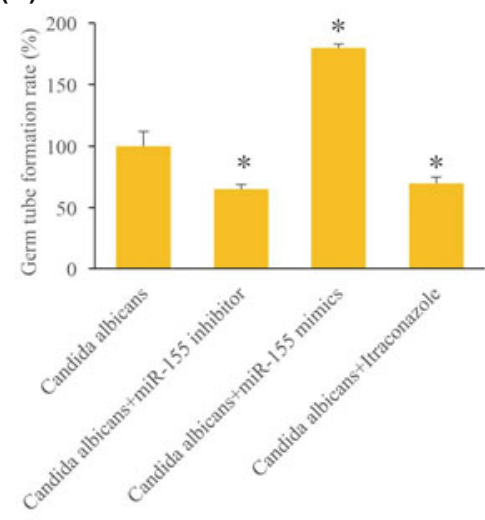

(B)

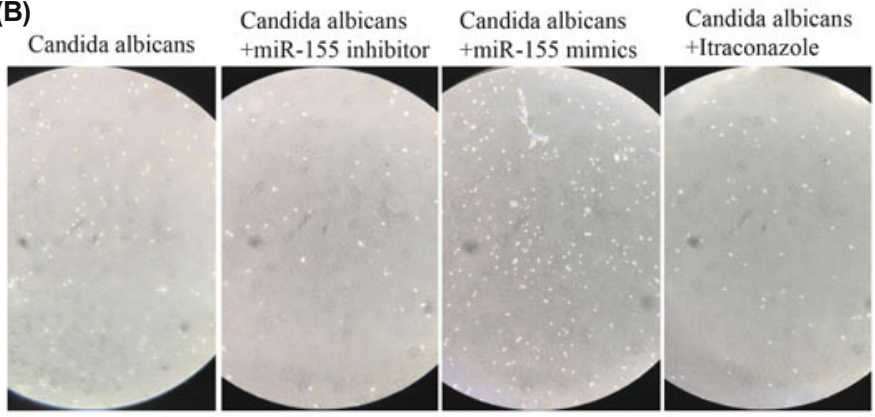

(D)
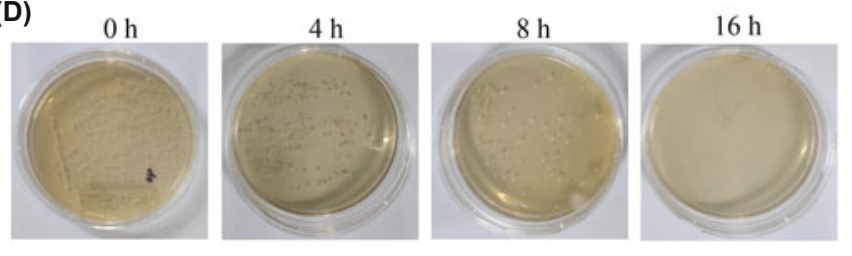

(E)

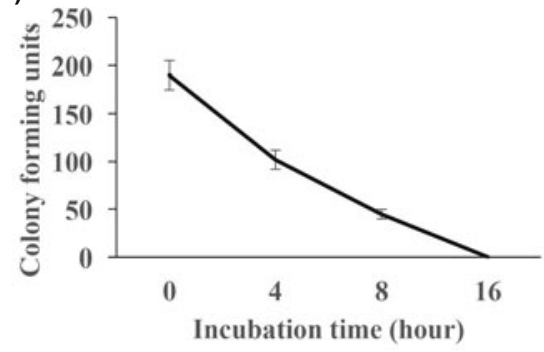

Fig. 1. Knockdown of miR-155 markedly inhibited the germ tube formation of Candida albicans. (A) miR-155 mimics and miR-155 inhibitor were constructed; (B) Influence of miR-155 on germ tube formation of C. albicans was investigated; (C) Influence of miR-155 on germ tube formation of C. albicans was analyzed; (D) Time-kill curves were conducted to investigate the influence of miR-155 on C. albicans; (E) The time-kill curve of miR-155 inhibitor in terms of C. albicans was observed. ${ }^{\star} P<0.05$ compared with group control or $C$. albicans. 


\section{Statistical analysis}

All results were presented as mean $\pm \mathrm{SD}$, and analyzed with SPSS software. Student's t-test was applied to compare findings between two groups. One-way ANOVA was applied to compare the findings among multiple groups, followed by Dunnett's t-test. $P<0.05$ was believed to be statistical difference.

\section{Results}

\section{Knockdown of miR-155 markedly inhibited the germ tube formation of $C$. albicans}

miR-155 mimics and miR-155 inhibitor were constructed (Fig. 1A) to investigate the influence of miR-155 on germ tube formation of C. albicans. We found that knockdown of miR-155 markedly decreased the germ tube formation of C. albicans, but transfection with miR-155 mimics greatly accelerated the growth rate of germ tube (Fig. 1B-C). In addition, the time-kill curve of miR-155 inhibitor in terms of $C$. albicans was detected. After $16 \mathrm{~h}$ incubation, total C. albicans was killed by treatment with miR-155 inhibitor (Fig. 1D-E).

\section{Influence of miR-155 on the acute lung injury induced by C. albicans}

The acute lung injury animal model was established through treatment with $C$. albicans. The alveolar septum in the group was increased greatly (Fig. 2A) and the bleeding area (Fig. 2B) was significantly enlarged compared with group control. However, transfection with miR-155 inhibitor could markedly could significantly suppress the damage caused by $C$. albicans, but overexpression of miR-155 greatly enlarged the damage of C. albicans (Fig. 2A-B). Treatment with Itraconazole also play a remarkable protection effect on lung tissue. In addition, we measured the levels of inflammatory factors in the serum after different treatments. The significant increased IL-10, TNF- $\alpha$, IL- 6 , and TNF- $\beta$ in the group $C$. albicans were markedly inhibited by miR-155 inhibitor and Itraconazole, but promoted by miR-155 mimics (Fig. 2C). The fungus load levels in different tissues and blood were also measured. Knockdown of miR-155 and administration with Itraconazole remarkably suppressed the fungus contents in lung, liver, kidney, and blood compared with group C. albicans (Fig. 2D).

(A)
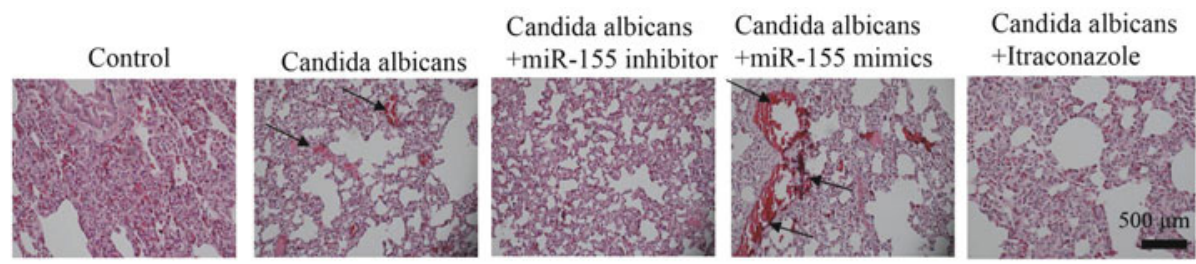

(B)

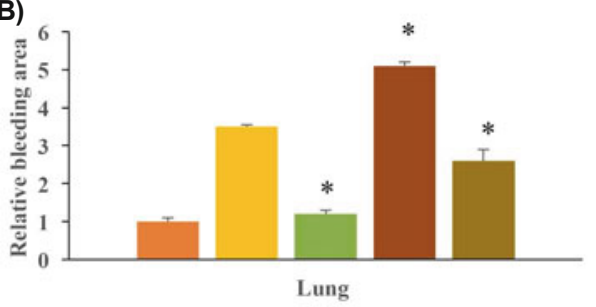

= Control

E Candida albicans

= Candida albicans+miR-155 inhibitor

- Candida albicans+miR-155 mimics

- Candida albicans+Itraconazole

(D)

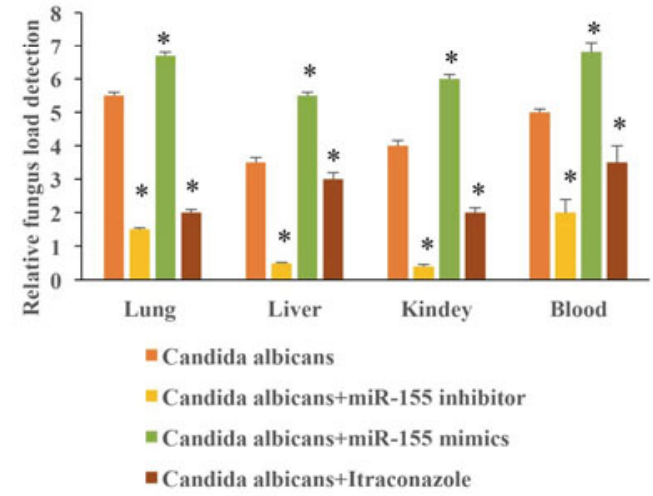

(C)

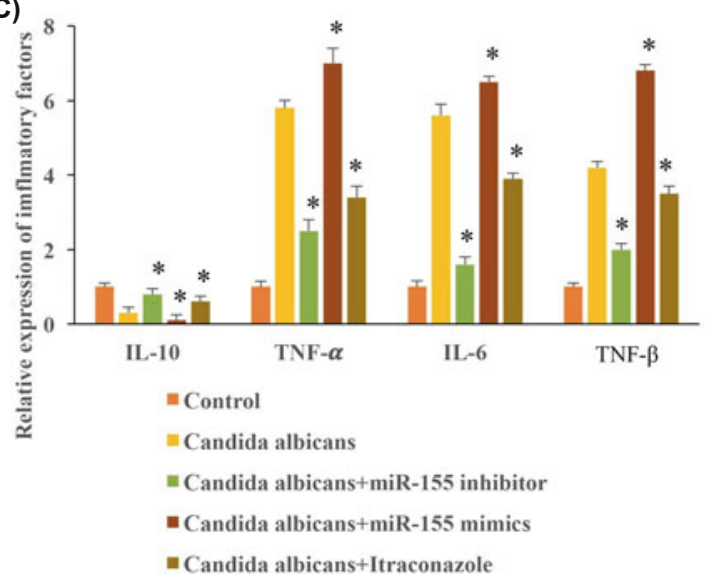

Fig. 2. Influence of miR-155 on the acute lung injury induced by Candida albicans. (A) Influence of miR-155 on the lung histological changes of acute lung injury animals; (B) Analysis of bleeding areas in the lung tissues; (C) The inflammatory factors in the serum were investigated; (D) The fungus load in different tissues were measured. ${ }^{\star} P<0.05$ compared with group $C$. albicans. Black arrows indicate the bleeding site. 
Influence of miR-155 on the expression of SOCS1 in the lung tissues and the binding site identification between miR-155 and SOCS1

The expression levels of SOCS1 in the lung tissues were detected. The level of SOCS1 was greatly suppressed in the group of C. albicans (Fig. 3A-C) compared with group control. However, the protein and mRNA levels of SOCS1 were greatly promoted by transfecting miR-155 inhibitor and treatment with Itraconazole compared with group C. albicans (Fig. 3A-C). The binding site between miR-155 and SOCS1 was also identified (Fig. 3D-E).
Knockdown of SOCS1 markedly reversed the influence of miR-155 inhibitor on the improvement of acute lung injury in vivo

The increased alveolar septum and bleeding areas in the lung tissues of group C. albicans were significantly improved after transfection with miR-155 inhibitor (Fig. 4A-B). However, sh-SOCS1 remarkably reversed the effect of miR-155 inhibitor, and aggravated the acute lung injury and bleeding in the lung tissue (Fig. 4A-B). In addition, the remarkable decrease of inflammatory factors and fungus load in the tissues induced by knocking down miR-155 were also reversed by

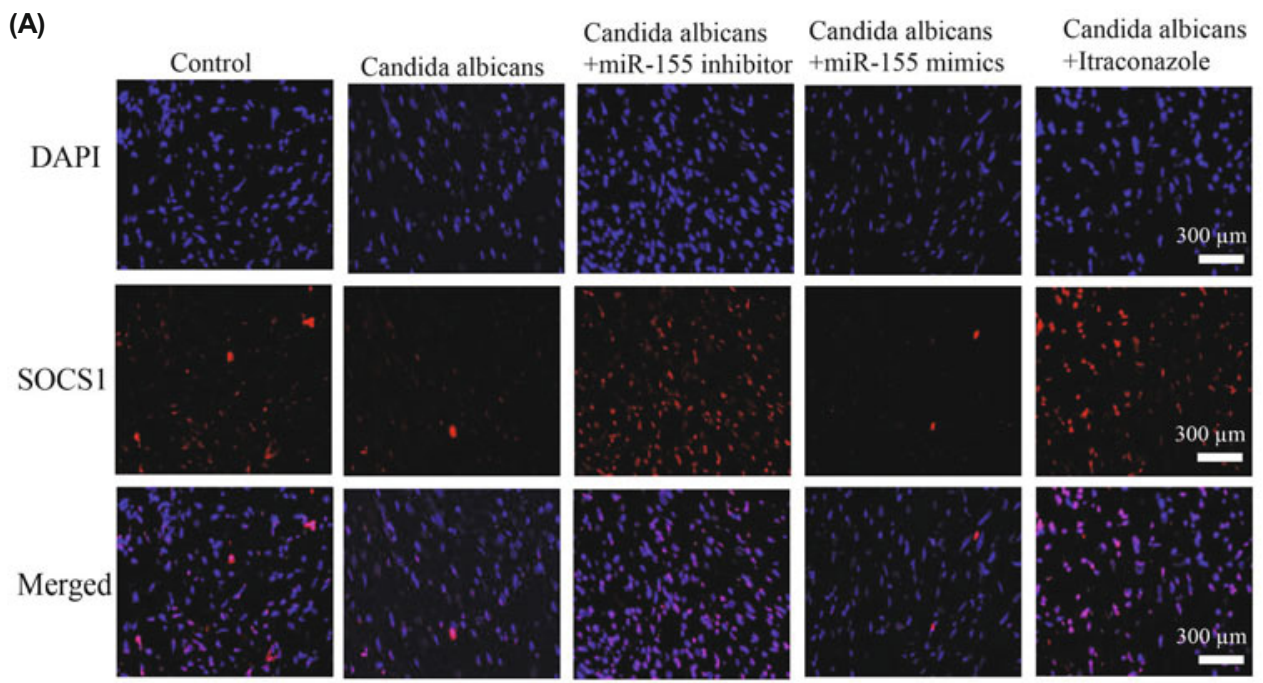

(B)

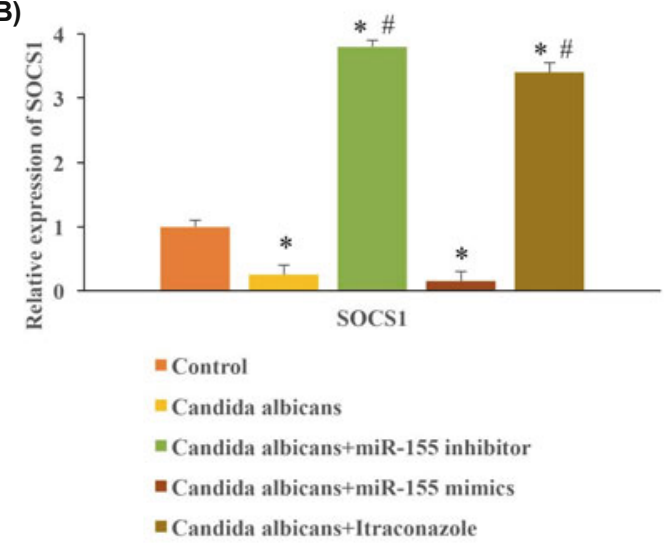

(D)

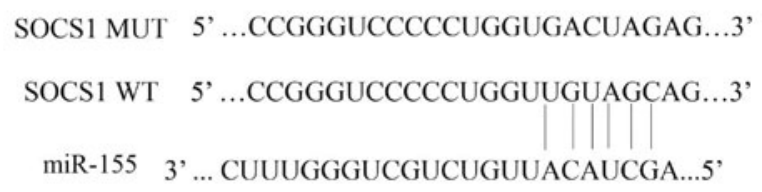

(C)

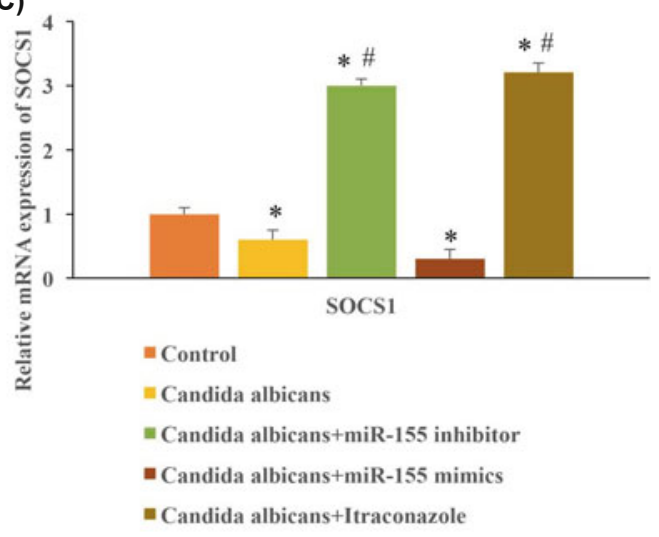

(E)

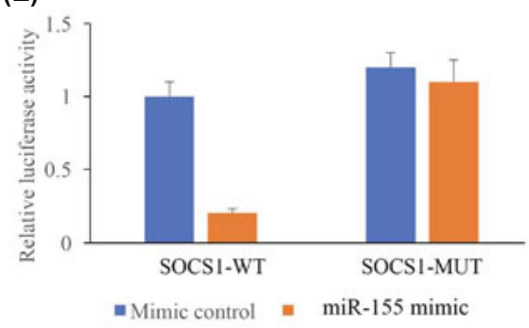

Fig. 3. Influence of miR-155 on the expression of SOCS1 in the lung tissues and the binding site identification between miR-155 and SOCS1. (A) The level of SOCS1 in the lung tissue was measured with IHC staining; (B) The level of SOCS1 in the lung tissue was analyzed; (C) The level of SOCS1 in the lung tissue was measured with RT-PCR assay; (D) The binding site between miR-155 and SOCS1 was predicted; (E) The binding site between miR-155 and SOCS1 was identified. ${ }^{*} P<0.05$ compared with group control, $\# P<0.05$ compared with group Candida albicans. 
sh-SOCS1 (Fig. 4C-D). We found that transfection with shSOCS1 greatly promoted the levels of inflammatory factors and fungus load in different tissues (Fig. 4C-D). In addition, we found that the decreased body weight of animals caused by $C$. albicans was significantly increased by miR-155 inhibitor. However, sh-SOCS1 markedly inhibited the influence of miR-155 inhibitor (Fig. 4E). Therefore, miR-155 might regulate acute lung injury through targeting SOCS1.

\section{Knockdown of SOCS1 greatly reversed the influence of miR- 155 inhibitor on the cell apoptosis in vitro}

The regulatory function between SOCS1 and miR-155 was also investigated in vitro. We found that cell apoptosis level in the group LPS + miR-155 inhibitor was remarkably decreased compared with group LPS (Fig. 5A-B). However, the influence of miR-155 inhibitor on cell apoptosis was greatly reversed by knocking down SOCS1 (Fig. 5A-B). Similar findings were observed in the mRNA expression of apoptosisrelated proteins. Transfection with miR-155 inhibitor significantly decreased the levels of Caspase- 3 and Bax, but increased Bcl-2 compared with group LPS (Fig. 5C). However, simultaneous administration with sh-SOCS1 markedly promoted the levels of Caspase- 3 and Bax, but suppressed Bcl-2 expression. Therefore, miR-155 might influence cell apoptosis through regulating SOCS1.

\section{Discussion}

Acute lung injury can further lead to acute respiratory distress syndrome (ARDS), which is one of the common causes
(A)

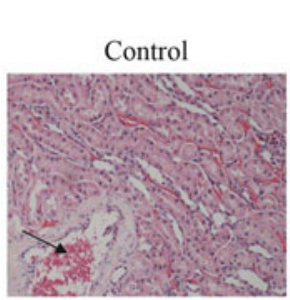

(B)

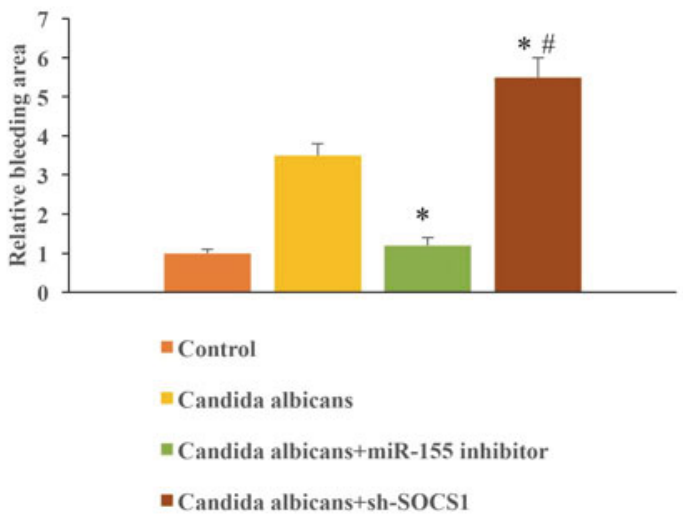

(D)

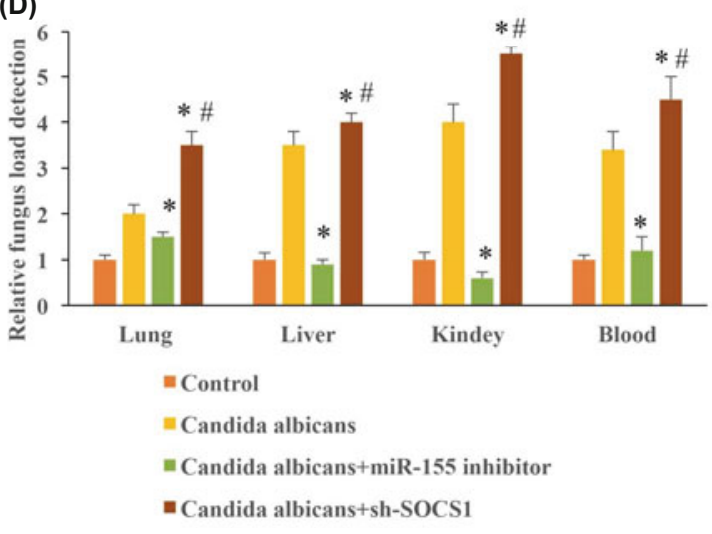

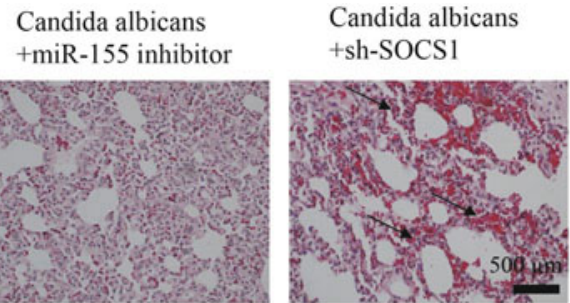

(C)

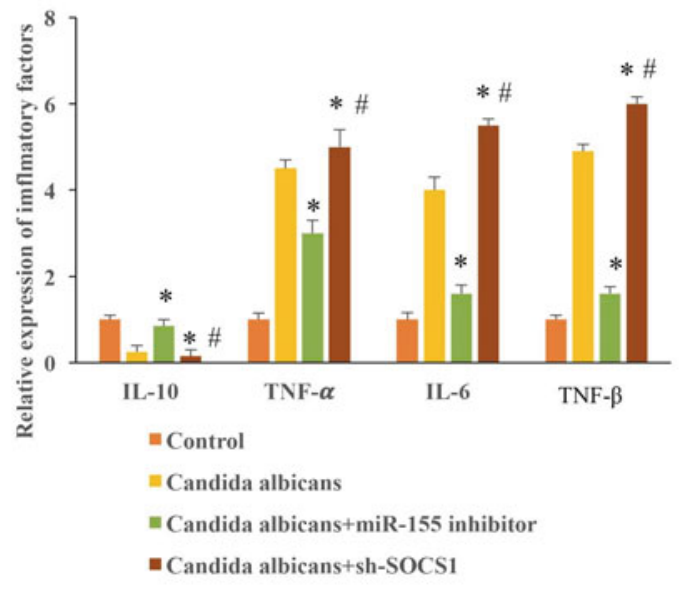

$(\mathrm{E})$

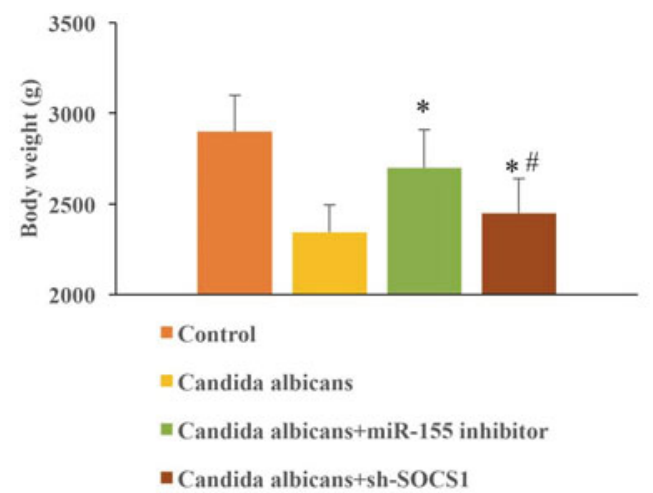

Fig. 4. Knockdown of SOCS1 markedly reversed the influence of miR-155 inhibitor on the improvement of acute lung injury in vivo. (A) Influence of SOCS1 on the lung histological changes of acute lung injury animals; (B) Analysis of bleeding areas in the lung tissues; (C) The inflammatory factors in the serum were investigated; (D) The fungus load in different tissues were measured; (E) The body weights of animals were analyzed. ${ }^{\star} P<0.05$ compared with group Candida albicans. ${ }^{\#} P<0.05$ compared with group C. albicans + miR-155 inhibitor. Black arrows indicate the bleeding site. 

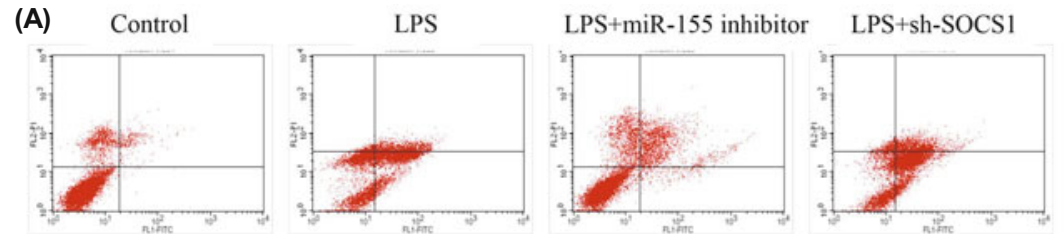

(B)

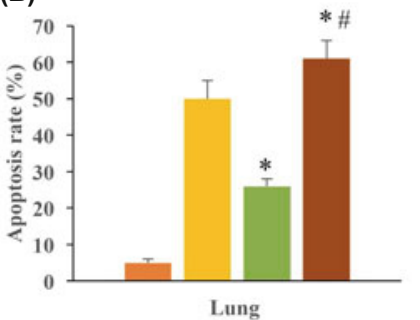

m Control

$=$ LPS

= LPS+miR-155 inhibitor

- LPS+miR-155 inhibitor+sh-SOCS1
(C)

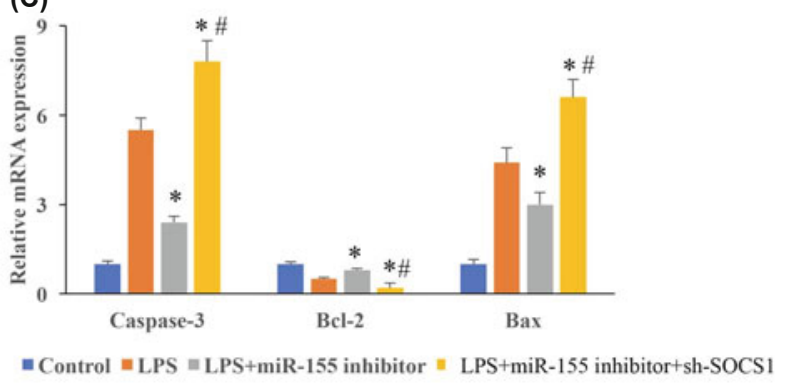

Fig. 5. Knockdown of SOCS1 greatly reversed the influence of miR-155 inhibitor on the cell apoptosis in vitro. (A) The influence of miR-155 inhibitor and sh-SOCS1 on cell apoptosis was measured; (B) The influence of miR-155 inhibitor and sh-SOCS1 on cell apoptosis was analyzed; (C) The influence of miR155 inhibitor and sh-SOCS1 on apoptosis-related protens was measured. ${ }^{\star} P$ $<0.05$ compared with group LPS, ${ }^{\sharp} P<$ 0.05 compared with group LPS + miR155 inhibitor. of death in critically ill patients (Deshpande and Zou, 2020). Acute lung injury is often induced by sepsis, surgical stress, inflammation, trauma, infection, and repeated massive blood transfusion (Shah et al., 2021). It is characterized by pulmonary edema, damage to alveolar epithelium and pulmonary capillaries, extensive inflammation of the lung, and often leads to shock and multiple organ failure (Li and Zou, 2020). At present, the main clinical treatment methods include comprehensive measures such as drug treatment and mechanical ventilation (Du et al., 2020). In recent years, gene targeted therapy has brought new ideas for the treatment of acute lung injury.

Candida albicans infection is a potential cause of acute lung injury. Several reports have indicated the regulatory role of miRNAs in C. albicans infection induced-diseases. miR-155 affected the macrophages infected by $C$. albicans through targeting Dectin-1 (Agustinho et al., 2017). miR-384-5p played a protective role in $C$. albicans induced acute lung injury. miR-384-5p contributed to C. albicans induced-acute lung injury via targeting PGC1 $\beta$ and enhancing the activation of the NF- $\kappa B, M A P K$, and Akt inflammatory signaling pathways (Yu et al., 2021). Knockdown of miR-204/miR-211 aggravated candidemia-induced kidney injuries through suppressing $\mathrm{Hmxl}$ and promoting immune-suppression (Li et al., 2014). Our findings that the improvement of acute lung injury by knockdown of miR-155 expand the understanding of the regulatory role of miRNAs in C. albicans induced diseases.

SOCS1 is known as an important inhibitor of immune response, and it could regulate inflammatory factors produced by immune cells (Su et al., 2009). SOCS1 plays a negative feedback role in cytokine signal transduction. The primary accession number of SOCS1 is O15524 (Juskevicius et al., 2017). Overexpression of SOCS1 inhibited IFN- $\gamma$ receptor phosphorylation and STAT1 activation (Liu et al., 2021). Several reports have suggested the protective role of SOCS1 in the pulmonary infectious diseases. Asthma is a typical Th2 type immune response. The production of Th2 type cytokines is significantly increased in patients with asthma. The high expression of SOCS1 in Th1 cells significantly inhibited IL-4/

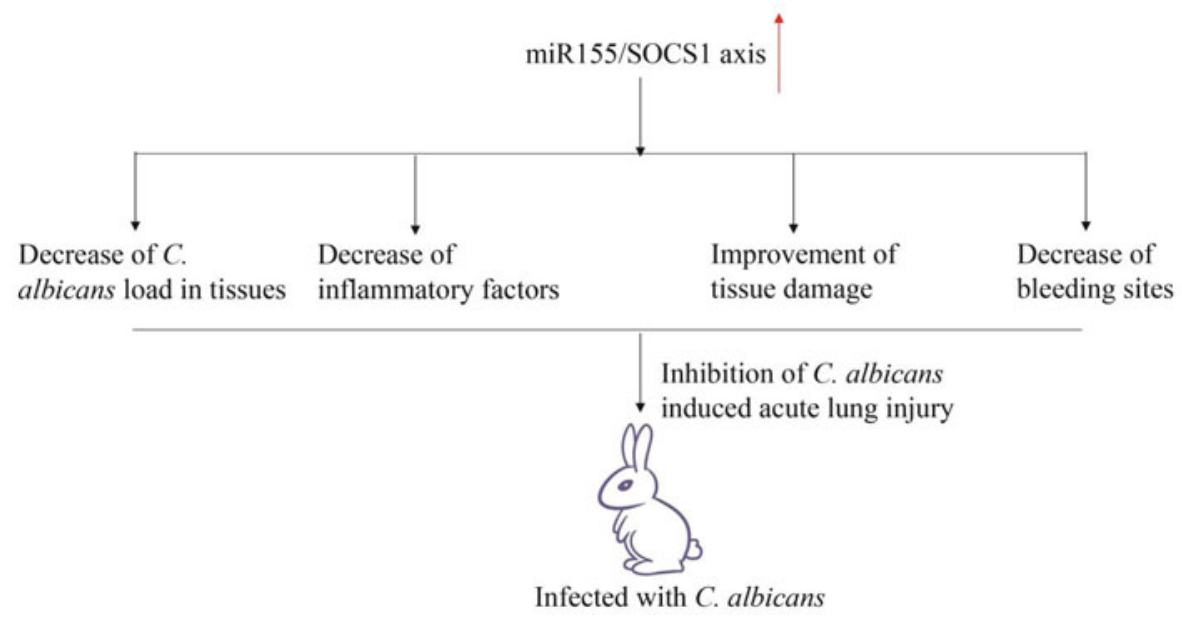

Fig. 6. The inhibition mechanism of miR-155/SOCS1 in the Candida albicans induced acute lung injury.

Infected with C. albicans 
STAT6 pathway, promoted the differentiation of Th1 type, and played a protective role in asthma (Dharajiya et al., 2009; de Oliveira et al., 2015).

The regulatory relationship between $\mathrm{miR}-155$ and SOCS1 has been proved in several types of diseases. miR-155/SOCS-1 signaling pathway is closely involved in the suppression of intracerebral hemorrhage-induced inflammation by glucocorticoid (Xu et al., 2016). miR-155 influenced the inflammation response via SOCS1 in atherogenesis (Yang et al., 2015; Ye et al., 2016). miR-155/SOCS-1 involved in the oxidized low-density lipoprotein induced inflammation inhibition by genistein (Zhang et al., 2017). Meanwhile, Propofol inhibited inflammatory response in LPS-activated microglia by through miR-155/SOCS1 pathway (Zheng et al., 2018). We demonstrated that knockdown of miR-155 suppressed $C$. albicans induced acute lung injury by activating SOCS1, which expand our understanding about the role of miR-155/ SOCS1 axis in disease (Fig. 6).

The limitation of this study is that only one strain of Candida was investigated. The effect of miR-155 on other Candida strains needs to be further studied to validate the conclusion of this research.

\section{Conclusion}

In summary, we proved that knock down of miR-155 could improve acute lung injury caused by $C$. albicans, suppressed inflammatory response, C. albicans infection, and cell apoptosis through targeting SOCS1. This study might provide a novel insight for the prevention and treatment of acute lung injury caused by C. albicans.

\section{Acknowledgements}

This study was supported by Natural Science Foundation of Fujian Province (2019J01536 and 2019J01538) and Fuzhou Science and Technology project (2021-S-wq1). We didn't receive any third-party support in conducting this research, analyzing the data, or preparing the manuscript for submission.

\section{Conflict of Interest}

The authors declare that they have no conflict of interest.

\section{Data Availability}

Data supporting this study has been presented in the manuscript, the data required by editor, reviewer and reader could be provided by the corresponding author.

\section{Author Contributions}

LX and YH conceived and designed the experiments; XL, QL, JL, TY, JX, and LC performed the experiments, XL and YG wrote the paper. All authors reviewed the manuscript.

\section{Ethical Statements}

Animal experiments were approved by the Animal Care and Use Committee of the Affiliated Fuzhou First Hospital of Fujian Medical University (20210014).

\section{Consent to Participate}

Not applicable.

\section{Consent for Publication}

All authors agree the publication of this manuscript.

\section{References}

Agustinho, D.P., de Oliveira, M.A., Tavares, A.H., Derengowski, L., Stolz, V., Guilhelmelli, F., Mortari, M.R., Kuchler, K., and SilvaPereira, I. 2017. Dectin-1 is required for miR155 upregulation in murine macrophages in response to Candida albicans. Virulence 8, 41-52.

Cheng, Y., Kuang, W., Hao, Y., Zhang, D., Lei, M., Du, L., Jiao, H., Zhang, X., and Wang, F. 2012. Downregulation of miR-27a* and miR-532-5p and upregulation of miR-146a and miR-155 in LPSinduced RAW264.7 macrophage cells. Inflammation 35, 13081313.

Chikuma, S., Kanamori, M., Mise-Omata, S., and Yoshimura, A. 2017. Suppressors of cytokine signaling, potential immune checkpoint molecules for cancer immunotherapy. Cancer Sci. 108, 574-580.

de Oliveira, J.R., Favarin, D.C., Tanaka, S.C., Balarin, M.A., Teixeira, D.N., Levy, B.D., and Rogério, A.D.P. 2015. AT-RvD1 modulates CCL- 2 and CXCL- 8 production and NF- $\kappa$ B, STAT-6, SOCS1, and SOCS3 expression on bronchial epithelial cells stimulated with IL-4. Biomed. Res. Int. 2015, 178369.

Deshpande, R. and Zou, C. 2020. Pseudomonas aeruginosa induced cell death in acute lung injury and acute respiratory distress syndrome. Int. J. Mol. Sci. 21, 5356.

Dharajiya, N., Vaidya, S., Sinha, M., Luxon, B., Boldogh, I., and Sur, S. 2009. Allergen challenge induces Ifng dependent GTPases in the lungs as part of a Th1 transcriptome response in a murine model of allergic asthma. PLoS ONE 4, e8172.

Du, J., Li, H., Lian, J., Zhu, X., Qiao, L., and Lin, J. 2020. Stem cell therapy, a potential approach for treatment of influenza virus and coronavirus-induced acute lung injury. Stem Cell Res. Ther. 11, 192.

Gamaletsou, M.N., Meletiadis, J., Chatziioannou, S., Panayiotides, I.G., Agadakos, E., Katsimpoulas, M., Kostomitsopoulos, N., Petraitis, V., Walsh, T.J., and Sipsas, N.V. 2019. Experimental Candida albicans osteomyelitis: microbiologic, antigenic, histologic, and ${ }^{18}$ FDG-PET-CT imaging characteristics in a newly established rabbit model. Med. Mycol. 57, 1011-1017.

Gingras, S., Parganas, E., de Pauw, A., Ihle, J.N., and Murray, P.J. 2004. Re-examination of the role of suppressor of cytokine signaling 1 SOCS1 in the regulation of toll-like receptor signaling. J. Biol. Chem. 279, 54702-54707.

Hassan, M.K., Waly, A.A., Elsayed, W., Keshk, S., Allam, W.R., and El-Khamisy, S.F. 2021. Integrative microRNA and gene expression analysis identifies new epigenetically regulated microRNAs mediating taxane resistance in ovarian cancer. Sci. Rep. 11, 562.

Juskevicius, D., Jucker, D., Klingbiel, D., Mamot, C., Dirnhofer, S., and Tzankov, A. 2017. Mutations of CREBBP and SOCS1 are independent prognostic factors in diffuse large B cell lymphoma, 
mutational analysis of the SAKK 38/07 prospective clinical trial cohort. J. Hematol. Oncol. 10, 70.

Kittelmann, S. and McGregor, A.P. 2019. Modulation and evolution of animal development through microRNA regulation of gene expression. Genes 10, 321.

Kobayashi-Sakamoto, M., Tamai, R., Isogai, E., and Kiyoura, Y. 2018. Gastrointestinal colonisation and systemic spread of Candida albicans in mice treated with antibiotics and prednisolone. Microb. Pathog. 117, 191-199.

Li, Q., Liu, J., Chen, M., Ma, K., Wang, T., Wu, D., Yan, G., Wang, C., and Shao, J. 2021. Abundance interaction in Candida albicans and Candida glabrata mixed biofilms under diverse conditions. Med. Mycol. 59, 158-167.

Li, X.Y., Zhang, K., Jiang, Z.Y., and Cai, L.H. 2014. MiR-204/miR-211 downregulation contributes to candidemia-induced kidney injuries via derepression of Hmxl expression. Life Sci. 102, 139-144.

Li, T. and Zou, C. 2020. The role of deubiquitinating enzymes in acute lung injury and acute respiratory distress syndrome. Int. J. Mol. Sci. 21, 4842.

Liu, H., Wang, W., and Liu, C. 2021. Increased expression of IFN- $\gamma$ in preeclampsia impairs human trophoblast invasion via a SOCS1/ JAK/STAT1 feedback loop. Exp. Ther. Med. 21, 112.

Mahernia, S., Hassanzadeh, M., Adib, M., Peytam, F., Haghighijoo, Z., Iraji, A., Mahdavi, M., Edraki, N., and Amanlou, M. 2021. The possible effect of microRNA-155 (miR-155) and BACE1 inhibitors in the memory of patients with down syndrome and Alzheimer's disease: design, synthesis, virtual screening, molecular modeling and biological evaluations. J. Biomol. Struct. Dyn. doi: 10.1080/07391102.2021.1873861.

Nidhi, P., Rolta, R., Kumar, V., Dev, K., and Sourirajan, A. 2020. Synergistic potential of Citrus aurantium L. essential oil with antibiotics against Candida albicans. J. Ethnopharmacol. 262, 113135.

Patterson, T.F., Fothergill, A.W., and Rinaldi, M.G. 1993. Efficacy of itraconazole solution in a rabbit model of invasive aspergillosis. Antimicrob. Agents Chemother. 37, 2307-2310.

Pena-Philippides, J.C., Caballero-Garrido, E., Lordkipanidze, T., and Roitbak, T. 2016. In vivo inhibition of miR-155 significantly alters post-stroke inflammatory response. J. Neuroinflammation 13, 287.

Shah, D., Das, P., Acharya, S., Agarwal, B., Christensen, D.J., Robertson, S.M., and Bhandari, V. 2021. Small immunomodulatory molecules as potential therapeutics in experimental murine models of acute lung injury (ALI)/acute respiratory distress syndrome (ARDS). Int. J. Mol. Sci. 22, 2573.

Su, L., Sun, Y., Ma, F., Lu, P., Huang, H., and Zhou, J. 2009. Progesterone inhibits Toll-like receptor 4-mediated innate immune response in macrophages by suppressing NF- $\kappa \mathrm{B}$ activation and enhancing SOCS1 expression. Immunol. Lett. 125, 151-155.

Sun, X., Sun, J., Shao, X., Feng, J., Yan, J., and Qin, Y. 2018. Inhibition of microRNA-155 modulates endotoxin tolerance by upregulating suppressor of cytokine signaling 1 in microglia. Exp. Ther. Med. 15, 4709-4716.

Sun, Q., Xiang, R.L., Yang, Y.L., Feng, K., Zhang, K., and Ding, W.Y. 2013. Suppressor of cytokine signaling 1 protects rat pancreatic islets from cytokine-induced apoptosis through Janus kinase/ signal transducers and activators of transcription pathway. Chin. Med. J. 126, 4048-4053.

Vila, T., Montelongo-Jauregui, D., Ahmed, H., Puthran, T., Sultan, A.S., and Jabra-Rizk, M.A. 2020. Comparative evaluations of the pathogenesis of Candida auris phenotypes and Candida albicans using clinically relevant murine models of infections. mSphere 5, e00760-20.

Wang, P., Hou, J., Lin, L., Wang, C., Liu, X., Li, D., Ma, F., Wang, Z., and Cao, X. 2010. Inducible microRNA-155 feedback promotes type I IFN signaling in antiviral innate immunity by targeting suppressor of cytokine signaling 1. J. Immunol. 185, 6226-6233.

Woodbury, M.E., Freilich, R.W., Cheng, C.J., Asai, H., Ikezu, S., Boucher, J.D., Slack, F., and Ikezu, T. 2015. miR-155 is essential for inflammation-induced hippocampal neurogenic dysfunction. J. Neurosci. 35, 9764-9781.

Xu, H.F., Fang, X.Y., Zhu, S.H., Xu, X.H., Zhang, Z.X., Wang, Z.F., Zhao, Z.Q., Ding, Y.J., and Tao, L.Y. 2016. Glucocorticoid treatment inhibits intracerebral hemorrhageinduced inflammation by targeting the microRNA155/SOCS1 signaling pathway. Mol. Med. Rep. 14, 3798-3804.

Xu, Y., Zhu, J., Feng, B., Lin, F., Zhou, J., Liu, J., Shi, X., Lu, X., Pan, Q., Yu, J., et al. 2021. Immunosuppressive effect of mesenchymal stem cells on lung and gut $\mathrm{CD} 8^{+} \mathrm{T}$ cells in lipopolysaccharideinduced acute lung injury in mice. Cell Prolif. 54, e13028.

Yan, C., Chen, J., Ding, Y., Zhou, Z., Li, B., Deng, C., Yuan, D., Zhang, Q., and Wang, X. 2021. The crucial role of PPAR $\gamma$-Egr-1-proinflammatory mediators axis in IgG immune complex-induced acute lung injury. Front. Immunol. 12, 634889.

Yang, Y., Yang, L., Liang, X., and Zhu, G. 2015. MicroRNA-155 promotes atherosclerosis inflammation via targeting SOCS1. Cell Physiol. Biochem. 36, 1371-1381.

Ye, J., Guo, R., Shi, Y., Qi, F., Guo, C., and Yang, L. 2016. miR-155 regulated inflammation response by the SOCS1-STAT3-PDCD4 axis in atherogenesis. Mediators Inflamm. 2016, 8060182.

Yu, D., Xu, C., Tu, H., Ye, A., and Wu, L. 2021. miR-384-5p regulates inflammation in Candida albicans-induced acute lung injury by downregulating PGC1 $\beta$ and enhancing the activation of Candida albicans-triggered signaling pathways. Sci. Prog. 104, 368504211014361.

Zhang, X., Hua, F., Yang, Z., Chen, Y., Teng, X., Huang, H., Zhao, Y., and Shen, Z. 2018. Enhancement of immunoregulatory function of modified bone marrow mesenchymal stem cells by targeting SOCS1. Biomed. Res. Int. 2018, 3530647.

Zhang, H., Zhao, Z., Pang, X., Yang, J., Yu, H., Zhang, Y., Zhou, H., and Zhao, J. 2017. Genistein protects against ox-LDL-induced inflammation through microRNA-155/SOCS1-mediated repression of NF- $\mathrm{KB}$ signaling pathway in HUVECs. Inflammation 40, $1450-1459$.

Zheng, X., Huang, H., Liu, J., Li, M., Liu, M., and Luo, T. 2018. Propofol attenuates inflammatory response in LPS-activated microglia by regulating the miR-155/SOCS1 pathway. Inflammation 41, 11-19. 